\title{
The Geometrical Regularization for Covering Irregular Bases with Karbandi
}

\author{
Amir Amjad Mohammadi ${ }^{1}$ - Maziar Asefi ${ }^{1}$ • \\ Ahad Nejad Ebrahimi ${ }^{1}$
}

\begin{abstract}
Karbandi is an arched covering pattern in Persian architecture which provides the interior of a structure with a particular geometric elegance and integrates architecture and structure in the creation of a meaningful spaces. The use of these structures is highly demanded in contemporary Persian architecture but requires a creative, modern design and construction strategy in order to meet new requirements, since traditional methods for implementing them causes them to be applicable only on regular bases. Here the authors investigate a solution to broaden their use over irregular geometry. This paper also aims at determining the possibility of administrating Karbandi geometry in the boundaries of an irregular polygonal shape. The result of this paper shows that it is possible to calculate and implement a Karbandi geometry in irregular bases when some specific conditions are met.
\end{abstract}

Keywords Karbandi · Karbandi geometry · Cross-ribbed vaulting · Vaults · Persian architecture $\cdot$ Islamic architecture

This paper has been extracted from a research-based design project (Modern Method of Construction) by master student: Amir Amjad Mohammadi at the Department of Architecture, Tabriz Islamic Art University, Tabriz, Iran, Under the Supervision of Dr. Maziar Asefi and Dr. Ahad Nejad Ebrahimi.

Maziar Asefi

masefi@tabriziau.ac.ir

Amir Amjad Mohammadi

a.amjad@tabriziau.ac.ir

Ahad Nejad Ebrahimi

ahadebrahimi@tabriziau.ac.ir

1 Tabriz Islamic Art University, Tabriz, Iran 


\section{Introduction}

This paper, by explaining Karbandi, one of the great achievement of Persian architecture, is aimed at increasing its usage in contemporary architecture. Karbandi is a decorative and geometrical covering element which is based on Islamic star patterns (Garofalo 2016). Islamic star patterns adorn buildings throughout the Islamic world (Kaplan and Salesin 2004). In Western countries they are known through the Alhambra Palace in Granada, Spain, one of the jewels of Islamic art (Irving 1931; Stewart 1974). Karbandi consists of intersecting ribs with a stellar geometry, which is one of the most common patterns ${ }^{1}$ in the restoration projects and new buildings of Persian architecture, including the Mausoleum of Omar Khayyam, Baba Tahir Hamadani, and others. However, due to certain geometrical and constructional limitations in the past, they have only been used over specific bases. ${ }^{2}$ However, little research has established the causes of these limitations and considered how they might be overcome so that Karbandi can be built over irregular bases. Understanding this fact and by considering the use of this structure in contemporary architecture, this paper aims at determining the possibility of administrating Karbandi geometry in the boundaries of a polygonal irregular base. This aim is firmly founded on the hypothesis that between a Karbandi geometry and its base there exists a precise and systematic connection and that by finding this connection it is possible to find a solution for using a Karbandi in irregular bases. The necessity underlying this research is to point out the dos and don'ts of Karbandi geometry in order to extend its usage in contemporary buildings. Drawing on what has been said, this research is going to develop a method of logical argumentation and data collection via direct observation, corpus analysis and computer modeling to search for answers for the following questions: (a) what are the required and adequate conditions for an execution of the geometry of an $n$-sided Karbandi on a variety of polygonal bases? (b) What qualities do the dimensions and geometry of the bases dictate to the geometry of Karbandi? (c) In what circumstances can a Karbandi geometry be used on irregular bases? ${ }^{3}$

\section{Literature Review}

There are various written resources on Karbandi, most of which are in Persian and are in descriptive and qualitative nature and are rarely address Karbandi's geometrical principles and the way it can be used in different geometries. The

\footnotetext{
${ }^{1}$ In Persian Architecture, Karbandi has been used for different purposes; in some cases, it has been used as a secondary roof under the main roof to bring a sense of geometric beauty and order into architectural spaces. In other cases, it appears at the stage of transferring a quadrilateral base to a dome and beautifully facilitates the transition. And in a few other cases, it has played the role of the main coverture of a space, and apart from the decorational effect, forms the main skeleton of the roof (Koliji 2012).

${ }^{2}$ For example, in the Historic Bazaar of Tabriz, wherever the base was irregular, another kind of covering patterns, such as Columbus vault, has been used.

3 The prerequisite for a complete understanding of what is offered in this research is a general familiarity with the Karbandi and basic principles of Euclidean geometry and some of its theorems. For such a familiarity, reference can be made to (Pour Ahmadi 2014).
} 


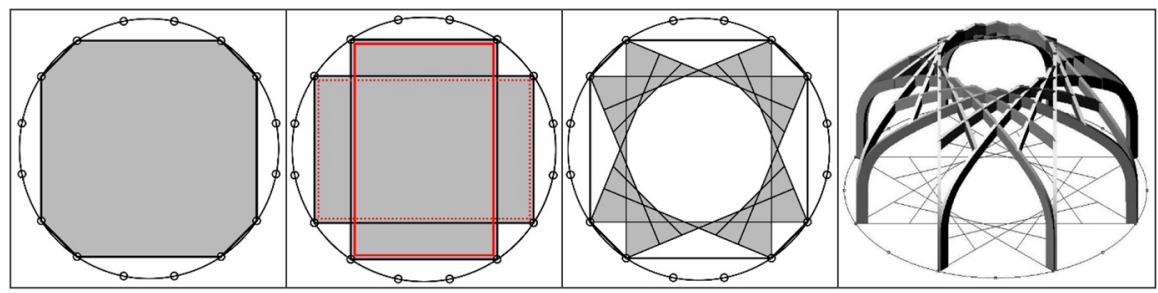

Fig. 1 A Karbandi on non-rectangular bases

quantitative approach taken in this research is built on a comprehensive interdisciplinary view towards the subject and discovering the effective factors of implementing Karbandi geometry on a variety of regular and irregular bases. The aim of this paper is to suggest a scholarly solution for the deployment of Karbandi geometry in polygonal irregular bases. The most salient resource in this regard is the book that has been written by Pirnia and Bozorgmehri (2006), in which, in addition to introducing the structural and drawing generalities of Karbandi, a traditional drawing method based on dividing a circle into equal sectors and drawing intersecting equal chords between the dividing points is presented. The formulas of this method are only applied for rectangular bases. In non-rectangular models, the base is initially assumed as a combination of two or more intersecting rectangles, then the geometry of Karbandi is drawn by the formulas of rectangular bases. Therefore, this method is actually applicable to rectangular bases and the bases created by intersecting two or more rectangles (Fig. 1 and Table 1).

Another traditional method for Karbandi development has been suggested by Sharbaf (2006). His method is for nimkar ${ }^{4}$ configurations for drawing Karbandi on symmetric bases without using a circumscribing circle but rather a circumscribing rectangle. Therefore, this method can only be used in rectangular and symmetric octagonal bases. There is also no formula in this method for calculating the number of sides of Karbandi, and drawing the Karbandi can only be possible by knowing the number of sides and the length of Karbandi's span. Lorzadeh (1979) in addition to explaining the executive points of Karbandi, has drawn several models in rectangular and symmetric octagonal bases which are quite similar to the method developed by Sharbaf. Moreover, in another research conducted by Mansour and Faramarzi (2011), in addition to shape-based and geometrical typology of different types of Karbandi, several equations were suggested for the development of the method proposed by Pirnia, but the equations are only for square and rectangular bases. Raeisi et al. (2013) have also explained a theoretical basis for Karbandi and their research was aimed at developing a correct knowledge of Karbandi's appearance. The research has identified three ambiguities (in naming, recognizing geometry and recognizing building function) in current models and presented a solution for each one. Accordingly, some criteria in building function, theoretical geometry and practical geometry have been proposed for distinguishing different cases.

\footnotetext{
4 A Karbandi which is constructed on an iwan, mehrab, etc. is called a nimkar (semi-work) because it produces just a half of the complete Karbandi and the front of the vault is open (Pour Ahmadi 2014).
} 
Table 1 Most common bases for Karbandi in Persian architecture

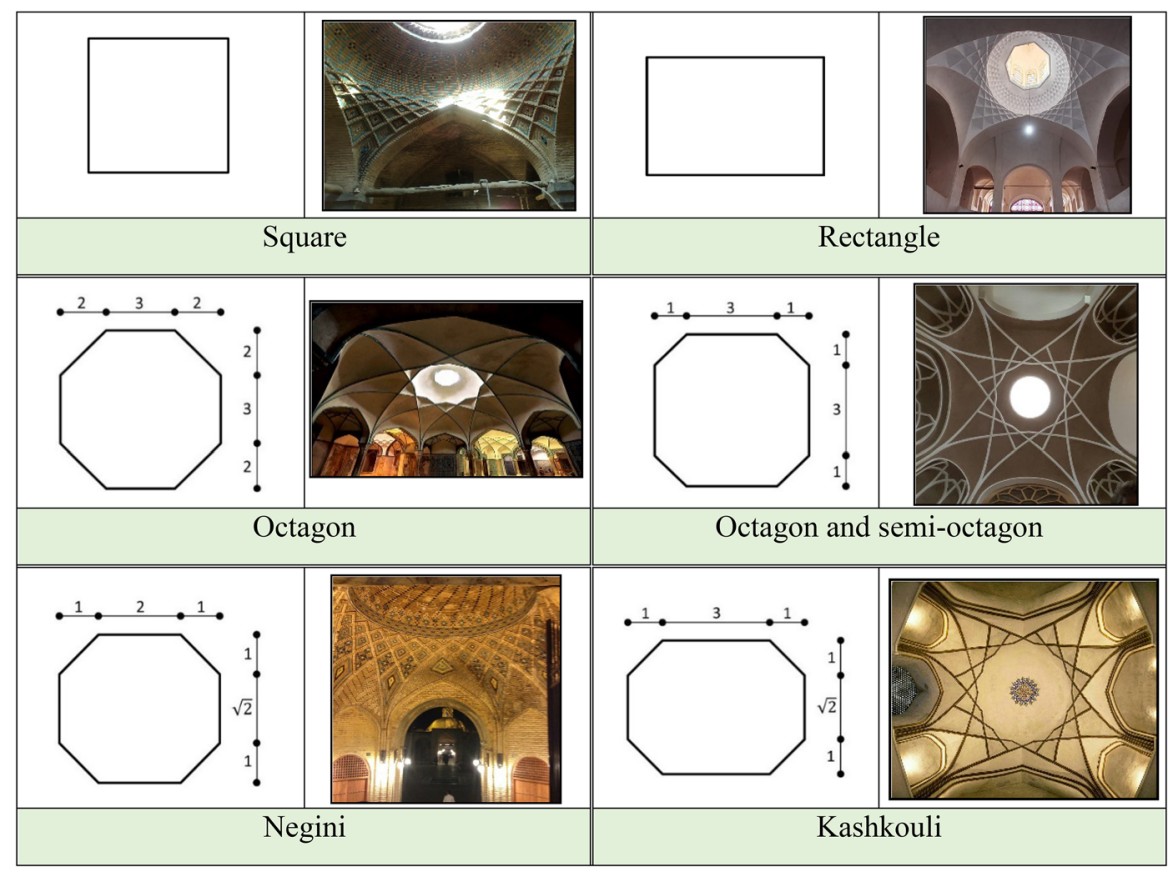

Finally, the most important and highly acclaimed feature of Karbandi is the correlation and coordination between its architectural and structural functions, which results in creating aesthetic and meaningful spaces. In this regard, the research carried out by Chenaghlou et al. (2017) in geometrical and structural behavior by Abaqus software confirms that this architectural configuration can be used as an efficient structure in terms of stability and weight. Therefore, using this type of system results in a good incorporation between architectural and structural aspects.

\section{Types of Karbandi}

A Karbandi system is divided generally into two categories: "Plumb" (shagooli in Farsi) and "out of plumb" (sar-seft in Farsi) (Sharbaf 2006; Memarian 2012). Out of plumb Karbandi have rarely been used in ancient Persian architecture (Pirnia and Bozorgmehri 2006). In order to better understand the Karbandi, these two types are explained as follows.

\section{Plumb Karbandi}

This category includes those types in which both parts of the rib arches that form Karbandi are placed in one plane and in line with each other (Memarian 2012: 346). Therefore, the rib arches connect to piers and directly transfer forces. In addition, a 


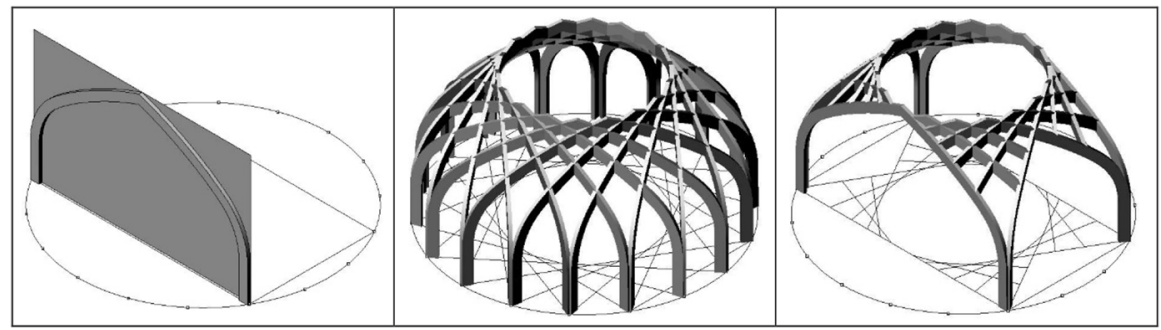

Fig. 2 Plumb Karbandi

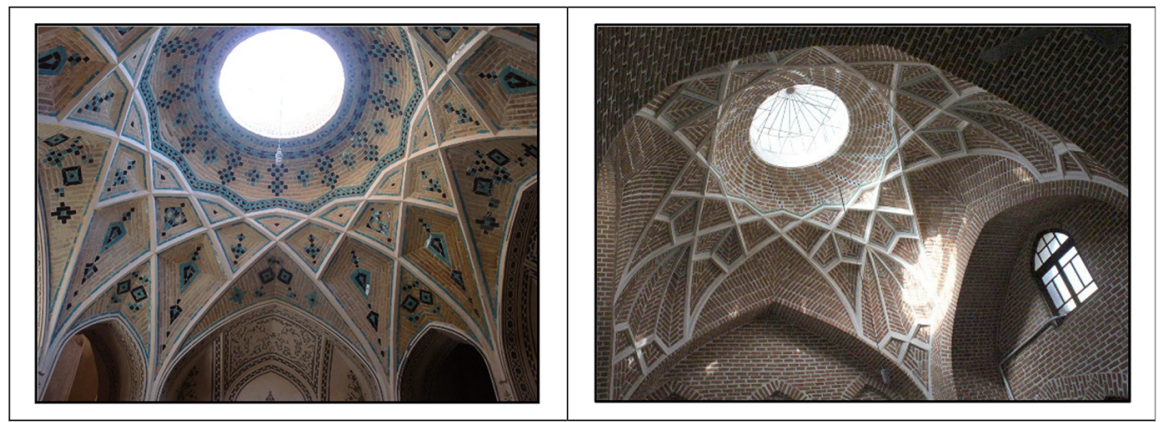

Fig. 3 Examples of Plumb Karbandi. a Left Men's bathroom of the Kashan Āmeri House, b right Haj Safar Ali Mosque in Tabriz

plumb Karbandi is vertical to the ground and presents a better load-bearing capability in comparison to an out of plumb Karbandi (Chenaghlou et al. 2017) (Fig. 2).

There are examples of this type of Karbandi in the men's bathroom of the Āmeri House in Kashan and the Haj-Safar-Ali Mosque in Tabriz (Fig. 3).

\section{Out of Plumb Karbandi}

If two parts of ribbed arches forming a Karbandi are not in the same plane, the resulting Karbandi is called "out of plumb Karbandi" (Memarian 2012). Since two sections of rib arches are not in line with each other, the rib arches cannot act as an efficient loadbearing structure in transferring loads. Therefore, it has less load-bearing capacity than a plumb Karbandi (Fig. 4). This type is used where it is required to limit the shamseh $^{5}$ boundary created on the very top of a Karbandi structure.

This type of Karbandi has been usually used on square bases, and an example of it can be seen in the Historic Bazaar of Tabriz (Fig. 5).

\footnotetext{
5 Literally "sun-like', it refers to sun-shaped element on the highest part of a Karbandi (see Fig. 10).
} 


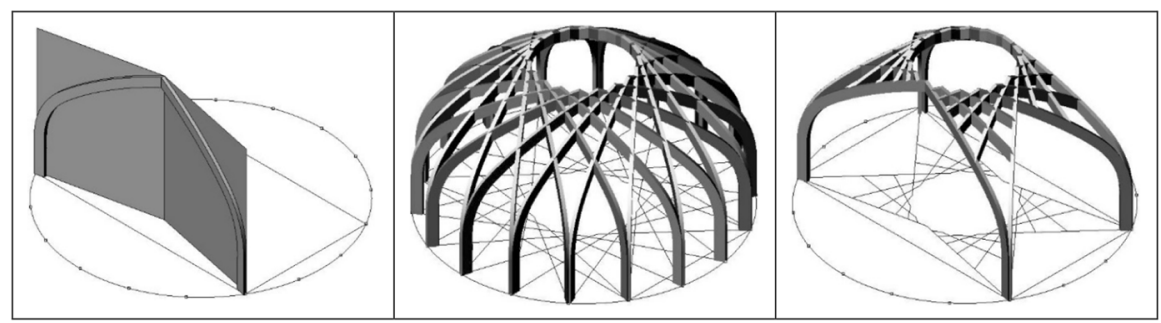

Fig. 4 Out of plumb Karbandi

\section{Common Bases for Karbandi}

The process of using traditional methods of drawing a Karbandi geometry offer little design flexibility in that they have a procedure only for applying Karbandi on regular bases. In other words, Karbandi have only been used for specific bases throughout the history of Persian architecture (Nava'I and Haji Qassemi 2011: 100). Regarding this issue, scholars (Helli 1986; Pirnia and Bozorgmehri 2006) have categorised the most common bases for Karbandi in traditional Persian architecture into the shapes of square, octagon, octagon and semi-octagon, negini (a type of octagon), and kashkouli (a type of octagon), as shown in Table 1.

In addition, there are a few cases of Karbandi that have been implemented on irregular bases. Where this has occurred, two types of solution have been commonly used. In the first solution, the base has been divided into regular and irregular parts and the Karbandi has been used only on the regular part. The irregular part has been also covered by two or more additional arch beams which have leant against Karbandi. An example of this type can be seen in the Malek-Carvansarai in the Historic Bazaar of Tabriz (Fig. 6).

In the second solution, in order to adapt Karbandi to the irregular base, some parts of a Karbandi have been cut and an incomplete model obtained which has been leant against a complete Karbandi and combined with it. There is an example of this type in the Aqa-Bozorg School-Mosque in Kashan (Fig. 7).

\section{Relations between Geometry and the Base of Karbandi}

Drawing a Karbandi is heavily dependent on establishing a precise relation between Karbandi geometry and the proportions and dimensions of its base. ${ }^{6}$ Such a relation mutually exists between Karbandi geometry and the base, in that the geometry of a Karbandi imposes a few limitations on the base and also takes some of its qualities from the proportions and dimensions of the base. In what follows, these relations and limitations are discussed and evaluated, and we propose specific regulations for implementing a Karbandi on regular and irregular bases.

\footnotetext{
${ }^{6}$ This present research is based on "Plumb Karbandi" excluding nimkar (semi-work).
} 

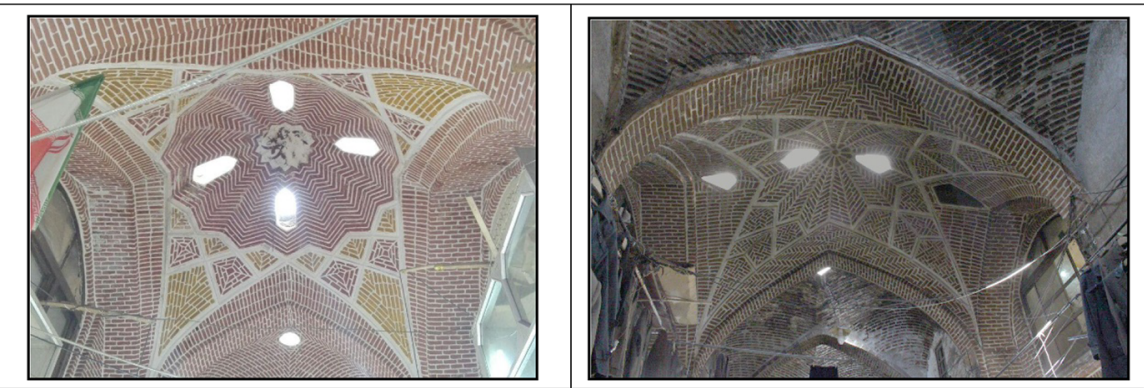

Fig. 5 Out of plumb Karbandi, the historic bazaar of Tabriz
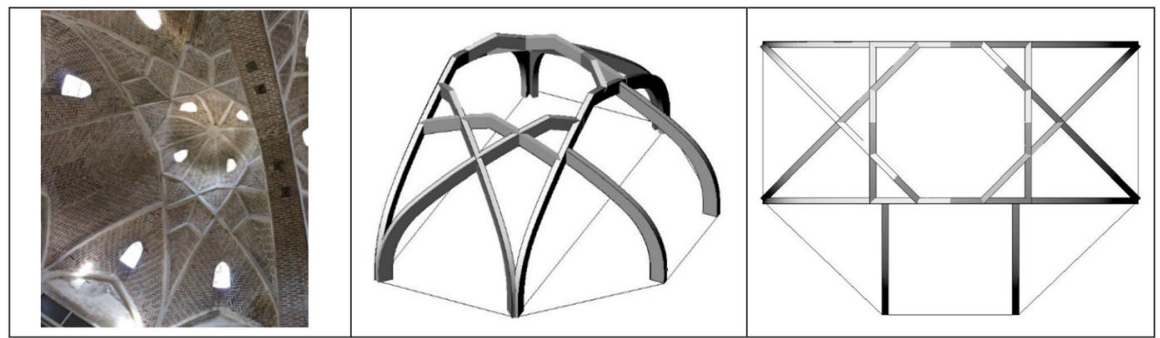

Fig. 6 Malek-Carvansarai in the Historic Bazaar of Tabriz

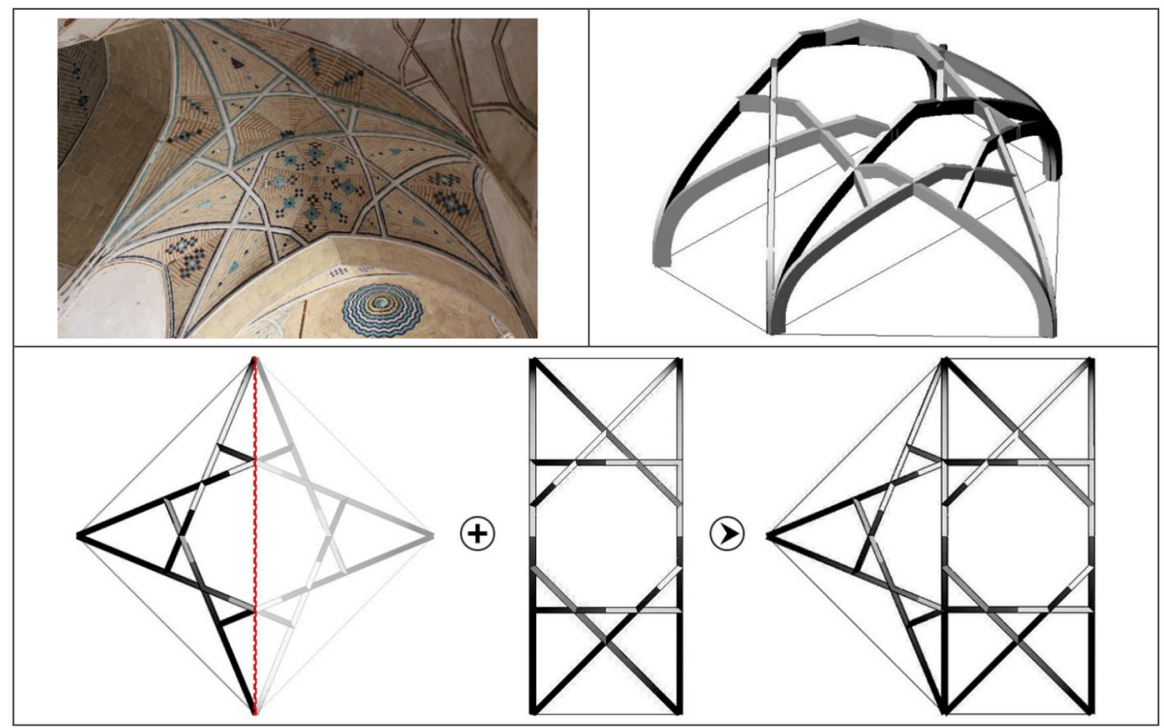

Fig. 7 Aqa-Bozorg School-Mosque in Kashan 


\section{Requirements for the Geometry of Karbandi}

The geometry of a Karbandi is the outcome of a totally exact drawing and mathematical methods, and for that reason some specific limitations and requirements are applied on its base. Analyzing samples of Karbandi within Persian architecture, and considering the findings of distinguished researchers such as Pirnia and Bozorgmehri (2006), and Nava'I and Haji Qassemi (2011) make it clear that the geometry of a Karbandi is based on three conditions. Unless these three principles are respected and satisfied, the result cannot be called 'Karbandi':

1. The plan of a Karbandi is based on dividing a circle into equal sectors and drawing intersecting equal chords between the dividing points; therefore, a circular part, called shamseh, is always formed inside a circumscribing circle;

2. The three-dimensional structure of a Karbandi is formed based on the rotation of an arch ribs round the center of the circumscribing circle. Therefore, the ribs of all the arches of a Karbandi share the same dimensions and proportions;

3. The center of the circumscribing circle to be placed inside the base.

In order to find a solution for drawing an $n$-sided Karbandi on irregular bases, we have analysed and evaluated the three conditions.

Condition 1 The most important issue in Karbandi drawing is the conformity of the drawing geometry pattern with the base. The first requirement for such conformity is for the base of Karbandi to be circumscribed by a circle. According to the principles mentioned, the geometry of a Karbandi should always be formed within the perimeter of its circumscribing circle (see Figs. 8, 9). Otherwise, by

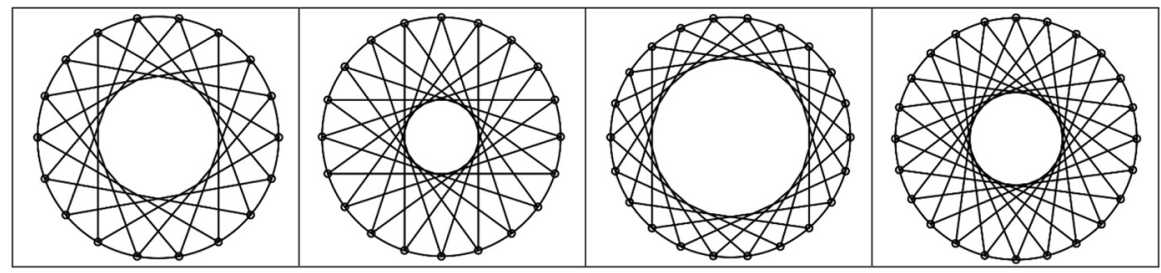

Fig. 8 Samples of bases which meet Condition 1

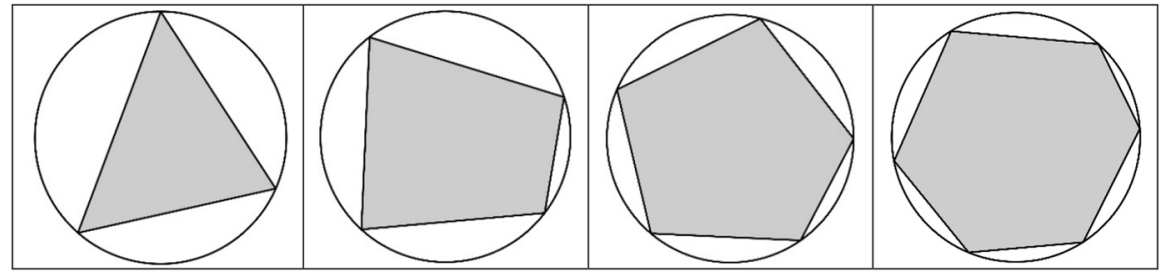

Fig. 9 The plan of a Karbandi 
illustrating the base on the geometry of Karbandi, one or more vertices of the base will not be positioned on the perimeter edge of the circle.

Condition 2 The second requirement for implementing the geometry of a Karbandi is the conformity of the vertices of the base with the dividing points of the circumscribing circle (Fig. 10).

If a base does not meet this requirement, there will be no choice but to divide the circumscribing circle into unequal sections, which results in drawing unequal chords. As a result, the outcome will contradict the principles of Karbandi geometry explained above and executing it will be impossible. For instance, in Table 2 there has been an attempt to draw a 16-sided Karbandi in a base which does not meet the second condition. As seen, due to the differences in their dimensions, the arch ribs

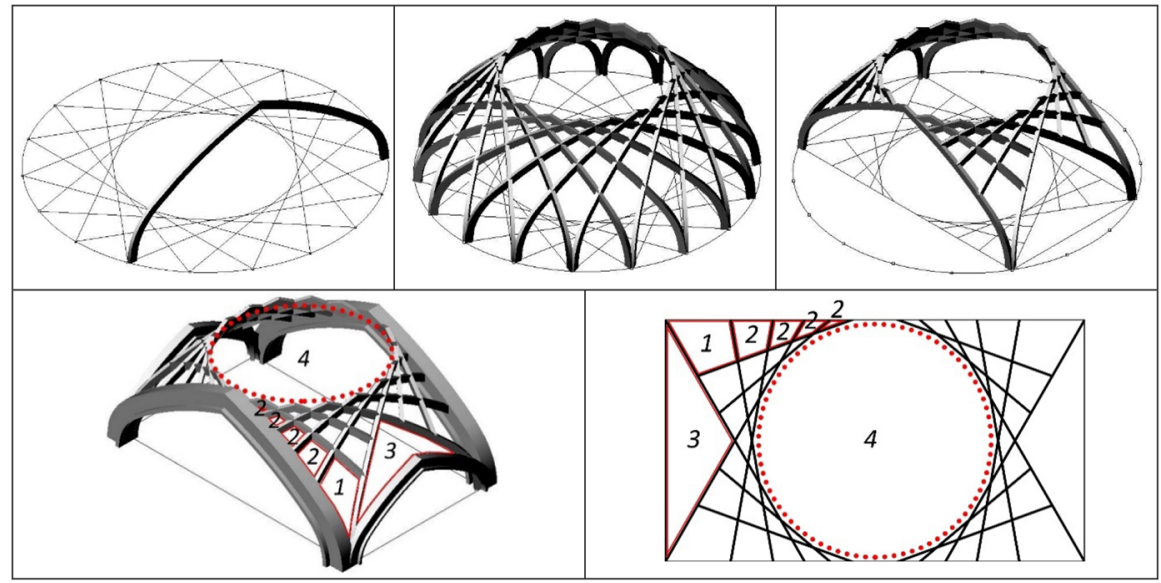

Fig. 10 Karbandi geometry shown in the third dimension: 1. Pa-barik, 2. Toranj, 3. Sousani, 4 Shamseh

Table 2 A demonstration of the inviability of implementing Karbandi on bases which do not meet Condition 2

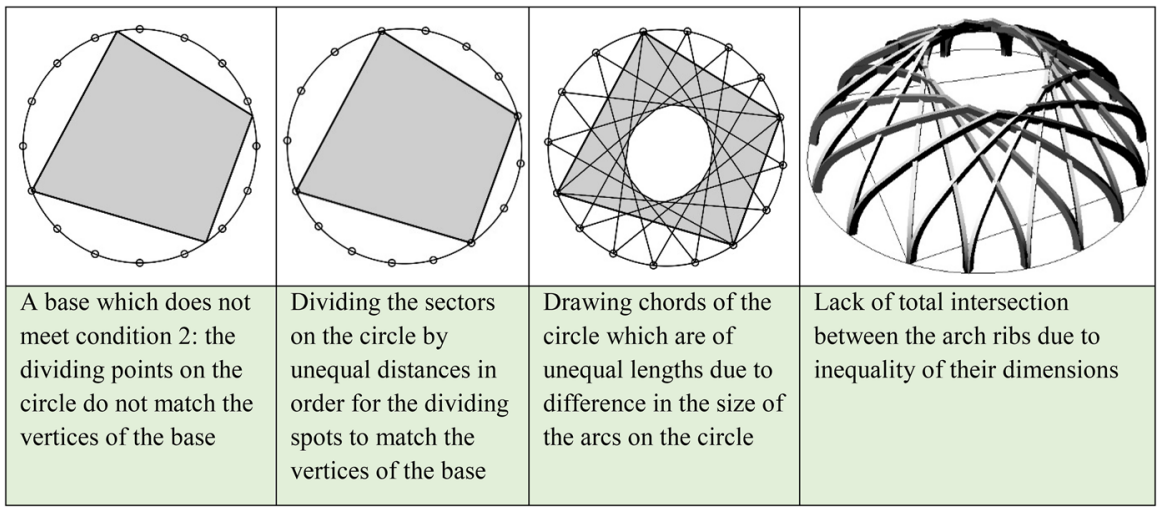


Table 3 Samples of bases which meet Condition 2

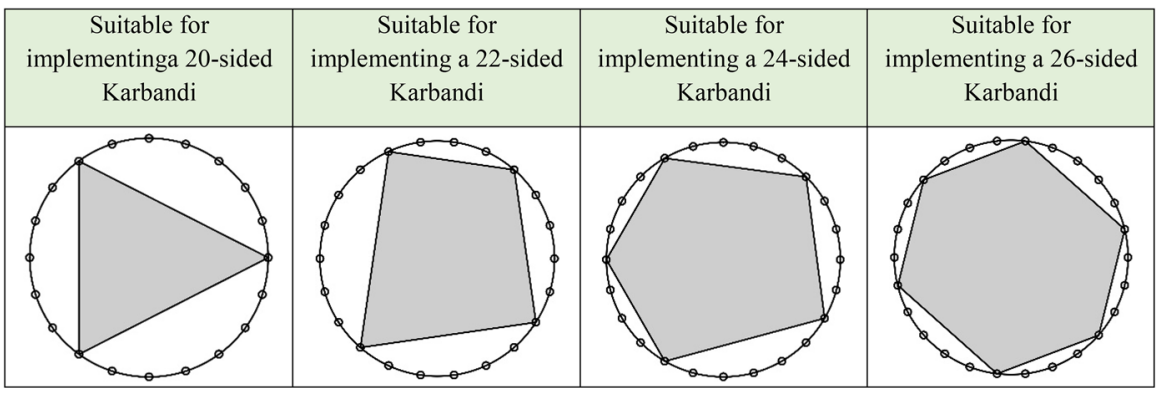

do not share a complete intersection, and some of them even overlap one another. It is obvious that the resulting configuration does not comply with the Karbandi principles as mentioned earlier.

It can be concluded that, an $n$-sided Karbandi is implementable only on bases where after dividing the circumscribing circle into $n$ equal arc, the vertices of the base are placed directly on the points at which the circle has been divided. Table 3 demonstrates samples of irregular trilateral, quadrilateral, pentagonal and hexagonal bases that, by complying with the aforementioned conditions, have created 20-, 22-, 24- and 26-sided Karbandi.

It is now required to find the properties of the four irregular shapes mentioned that can be used as a base for creating a Karbandi geometry. To do so, every vertex of the base is connected to the center of the circumscribing circle and therefore a number of central angles are formed. As seen in Table 4, an arc facing each of the central angles is a multiple of the sectors formed on the circle. For example, in the trilateral base, given that the circumscribing circle has been divided into twenty equal sectors, the angle facing each sector equals $360 / 20=18$. Therefore, every arc facing the central angles is a multiple of $18^{\circ}$. It can thus be concluded that a 20 -sided Karbandi is implementable in bases in which the central angles formed by connecting the verities of the base to the center of the circumscribing circle, are all a multiple of $18^{\circ}$. Table 4 shows the above quality for 22- to -26-sided Karbandi.

Now it is possible to extend the latter point to the base of $n$-sided Karbandi. Accordingly, the second condition for implementing an $n$-sided Karbandi in a variety of bases is that the angles formed as a result of connecting the vertices of the base to the center of the circle to be a multiple of $360^{\circ}$ divided by the number of the sides of the Karbandi (Eq. 1).

$$
\hat{a}, \hat{b}, \hat{c}, \hat{d}, \ldots\left(\frac{360^{\circ}}{n}\right) x, x \in N \text {. }
$$

Condition 3 The third condition for implementing a Karbandi geometry is that the center of the circumscribing circle must be placed inside the base. If this requirement is not met, the shamseh of Karbandi will, geometrically, be formed outside the base, and the base will only include part of the Karbandi geometry. 
Table 4 Attributes of bases suitable for implementing 20-, 22-, 24- and 26-sided Karbandi

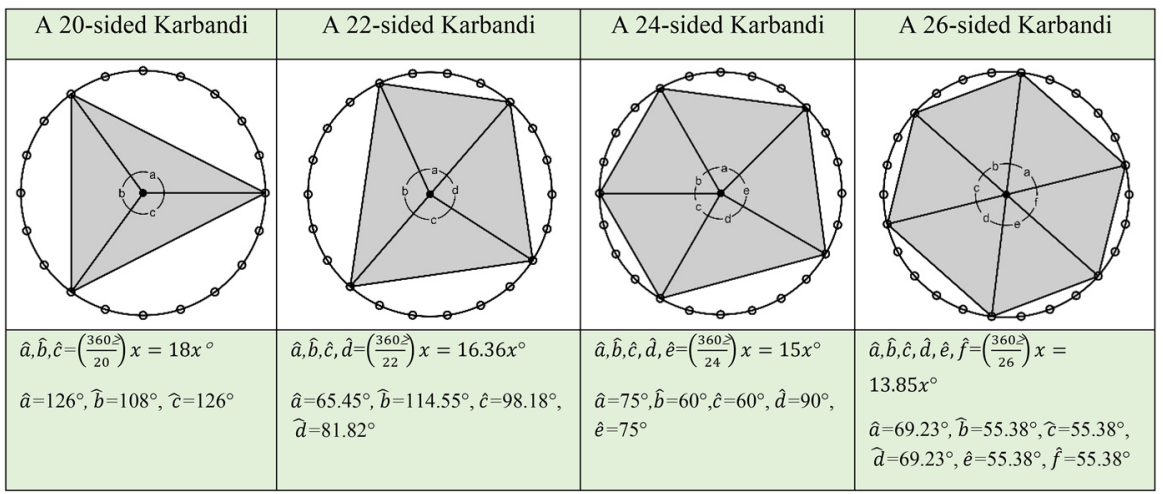

Table 5 Condition 3 for implementing Karbandi geometry

Samples of bases which meet condition 3
(viability of implementing Karbandi on these bases)
(inviability of implementing Karbandi on these
bases)

Therefore, implementing a Karbandi will not be possible (Table 5). In other words, as it is not possible to draw more than one circle inscribing a polygon, if the third condition is not met, it will be impossible to bring the shamseh of Karbandi inside the base.

\section{Requirements for the Base}

The implementation of a Karbandi geometry on any base will have unique requirements which are precisely related to the dimensions and proportions of the base at hand. This section seeks to point out a number of relations through which it 
is possible to choose and draw an appropriate Karbandi for every base. Here the most important factors determined by the base are:

- the number by which the dividing points on the circle will be connected to each other (e.g., 2 by 2,3 by 3 , etc.);

- the number of the sides of Karbandi.

Rules for connecting the dividing points of the circle (e.g., 2 by 2, 3 by 3, etc.) As pointed out earlier, the geometry of Karbandi is based on dividing the circumscribing circle into equal sectors and drawing intersecting chords between the dividing points. However, connecting the dividing points does not occur by optional distances. In rectangular bases, the longest side of the base is the decisive factor. That is, if the length of the rectangle faces $k$ sectors of the circle, all the dividing points on the circle will be connected to each other by a distance of $k$ by $k$ (Pirnia and Bozorgmehri 2006: 13). This strategy can also be applicable for irregular bases. However, it should be noted that if on irregular bases, as on rectangular bases, the dividing points on the circle are joined $k$ by $k$ (where $k$ is the number of the sectors of the circle facing the longest side of the base), in some outer parts of the Karbandi the sousani will be removed (Table 6), and consequently the consistency and the proportion of the components will be affected. To avoid this, we can connect the dividing spots by a distance of $k+1$ by $k+1$ (Table 8). After connecting the dividing points on the circumscribing circle, we erase all the lines which are protracted out of the base or the ones placed inside the sousani in order to create the shape of Karbandi.

As apparent in Table 6, if the longest side of an irregular base faces $k$ sectors of the circumscribing circle, the number by which the dividing points on the circle will be connected to each other can be obtained through Eq. (2):

$$
D=k+1 \text {. }
$$

It should be noted that Eq. (2) is not always applicable. If the longest side of the base is too long so that by dividing points by a distance of $k+1$ by $k+1$, all the chords drawn afterwards will cross the center of the circle, the shamseh of the Karbandi is removed (Table 7). In such bases, the number by which the dividing points on the circle will be connected to each other is obtained through Eq. (3):

$$
D=k \text {. }
$$

This issue mostly occurs in Karbandi with a low number of sides. As the number of the sides of Karbandi increases, the possibility of encountering this problem decreases.

\section{Number of the sides of Karbandi}

The number of the sides of a Karbandi is not arbitrarily selected, but rather the dimensions of the base on which a Karbandi is to be located determines the type of Karbandi (Pirnia and Bozorgmehri 2006: 12). For instance, in some cases, due to some structural and static considerations, along with increasing the dimensions of 
Table 6 Comparing two types of connecting the dividing points on the circle with a distance of $K$ by $K$ and $K+1$ by $K+1$

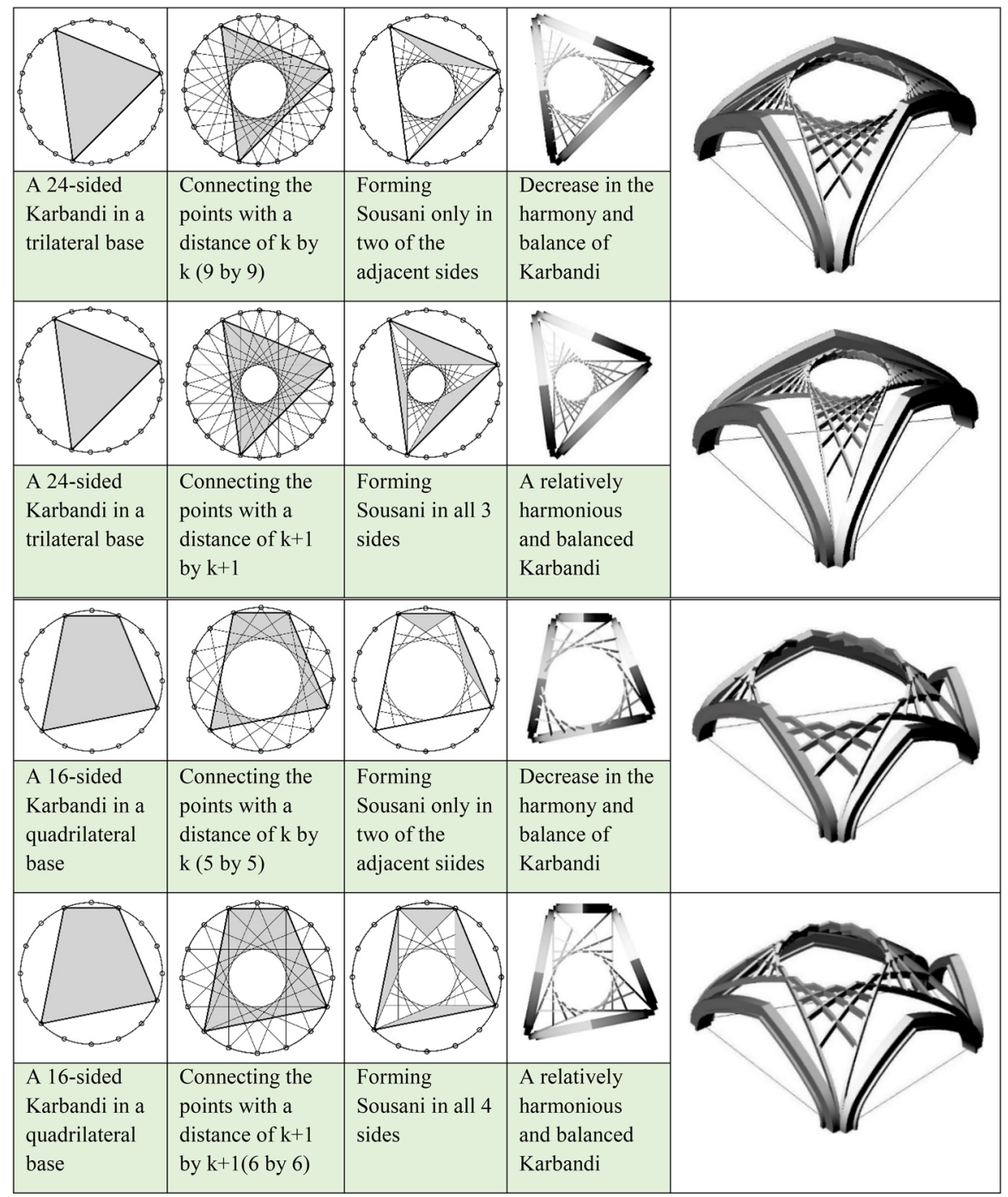

the base, the number of the sides of our Karbandi will have to be increased in order for the weight of the roof to be shared by more ribs. For rectangular bases Eq. (4) has been suggested for determining the sides of a Karbandi (Pirnia and Bozorgmehri 2006: 12).

$$
n=2(a+b-2)=P-4,
$$

where variable $a$ and $b$ are the numbers for the length and width of the rectangle respectively, $P$ is the base perimeter and $n$ is the suggested number of sides for the 
Table 7 Two samples of the specific type for irregular bases where the dividing points on the circle needs to be connected with a distance of $K$ by $K$

\begin{tabular}{l|l|l|l}
$\begin{array}{l}\text { Sample of the particular } \\
\text { types of trilateral and } \\
\text { quadrilateral bases }\end{array}$ & $\begin{array}{l}\text { Connecting the dividing } \\
\text { points with a distance of } \\
\mathrm{k}+1 \text { by } \mathrm{k}+1(7 \text { by } 7) \text { and } \\
\text { failing at forming a } \\
\text { Karbandi geometry }\end{array}$ & $\begin{array}{l}\text { Connecting the dividing } \\
\text { points with a distance of } \\
\mathrm{k} \text { by } \mathrm{k}(6 \text { by } 6) \text { and } \\
\text { forming a Karbandi } \\
\text { Geometry }\end{array}$ & $\begin{array}{l}\text { Geometry of a 14-sided } \\
\text { Karbandi }\end{array}$
\end{tabular}

rectangular Karbandi. Additionally, since the number of the sides of a Karbandi is always an even natural number, Eq. (5) is an extension of Eq. (4) for non-rectangular bases.

$$
n=\left\{\begin{array}{c}
{[P-4]+1=[P]-3, \quad[P] \in \text { Odd }} \\
{[P-4]=[P]-4, \quad[P] \in \text { Even }}
\end{array}(P \text { is the perimeter of the base }) .\right.
$$

\section{Karbandi on Irregular Bases Which Meet the Three Conditions}

As mentioned earlier, to accurately implement an $n$-sided Karbandi on a polygonal base, the aforementioned three conditions should be met. Table 8 shows examples of 20 to 24-sided Karbandi drawn according to the mentioned principles and strategies.

According to the examples shown in Table 8, it is evident that on any surrounded polygonal base, if the angles formed by connecting the vertices of the base to the center of the circumscribing circle are a multiple of $18^{\circ}$, a 20 -sided Karbandi is implementable. If they are a multiple of $16.36^{\circ}$, a 22-sided Karbandi is implementable. And overall, if they are a multiple of $\frac{360^{\circ}}{n}$, an $n$-sided Karbandi is formed. However, given the fact that the number of the sides of a Karbandi is invariably dependent on the perimeter of the base (Eq. 5), it is rare for the number of the sides of Karbandi bases which have been acquired via Eq. (5) to precisely match the quality calculated via Eq. (1). Consequently, Condition 2 does not hold for most bases. Keeping this fact in mind, the following section will seek to come up with a method through which it is possible to calculate and draw a Karbandi 
Table 8 Samples of Karbandi in bases which meet all three conditions

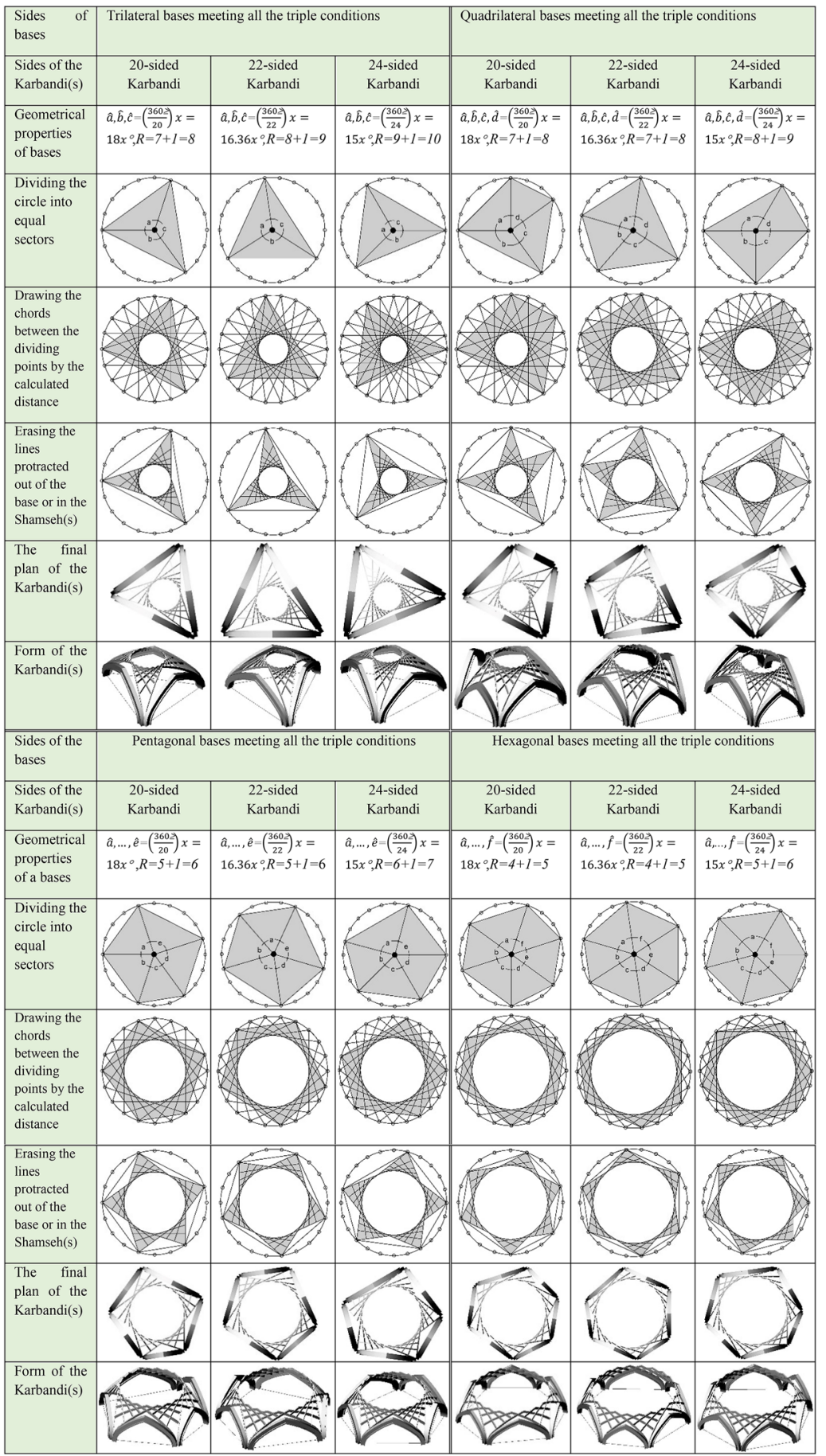


geometry for such bases which do not meet Condition 2, with the lowest level of error.

\section{Karbandi on Irregular Bases (Meeting Conditions 1 and 3)}

As explained earlier, implementing a Karbandi geometry in a totally exact way is not possible for many types of geometrical bases. Therefore, to create a Karbandi in those geometries, it is necessary to reconfigure the existing base until it meet the Condition 2 and the design requirements. By doing that, a Karbandi can be created with the least amount of error. This process has already been carried out by traditional architects in order to come to a proper Karbandi for the required geometry. They were aware of the fact that the calculated proportions of a Karbandi for a certain base-even a rectangular one-does not usually precisely match the dimensions of the base. So, at the implementing stage, they adjust the geometry of Karbandi to the base by slightly transposing the edges, a process which is called Kast-Afzoud (decreasing-increasing) (Pirnia and Bozorgmehri 2006: 12). With the latter fact in mind, and through a few stages, we propose a method through which it is possible to implement a Karbandi in any surrounded polygon with the lowest level of error.

\section{Stage 1}

For a better demonstration of this method, this part of the paper will propose an innovative drawing method in a number of randomly selected polygons (Table 9). At the first stage, we acquire the perimeter of the bases and calculate the appropriate number of sides for Karbandi using Eq. (5) (Table 9).

Example of triangular shape:

$$
\begin{gathered}
{[P]=[9.13+8.60+8.16]=[25.89]=25,} \\
n=\left\{\begin{array}{l}
{[P]-3, \quad[P] \in \text { Odd }} \\
{[P]-4, \quad[P] \in \text { Even }}
\end{array} \Rightarrow N=[P]-3=22 .\right.
\end{gathered}
$$

As obtained for the above equation, the appropriate Karbandi for the dimensions of the proposed triangle is a 22-sided one. The appropriate number of sides for Karbandi on 4-sided (quadrilateral), 5-sided (pentagonal), and 8-sided (octagonal) according to the same equation are 24, 26 and 26, respectively (Table 11).

\section{Stage 2}

In the second stage, the vertices of the base are connected to the center of the circumscribing circle and the formed angles are calculated (Table 9). According to Condition 2 (Eq. 1), all the angles need to be a multiple of the quotient of dividing $360^{\circ}$ by the number of the sides of Karbandi. Therefore, to find the polygon which is the closest one to the base in hand that does meet Condition 2, we need to find the closest angles to the central angles of the base, which are a multiple of $\left(\frac{360^{\circ}}{n} x\right)$. 
Table 9 Drawing a few randomly surrounded bases and measuring their sides and central angles

Random trilateral

$$
\begin{gathered}
\hat{a}, \hat{b}, \hat{c}=\left(\frac{360^{\circ}}{22}\right) x=16.364, x \in N \\
\hat{a}=132.02^{\circ}, \quad \frac{132.02^{\circ}}{16.364^{\circ}}=8.07 \Rightarrow \hat{a} \approx 8 \times 16.364^{\circ}=130.91^{\circ} \\
\hat{b}=118.65^{\circ}, \quad \frac{118.65^{\circ}}{16.364^{\circ}}=7.25 \Rightarrow \hat{b} \approx 7 \times 16.364^{\circ}=114.55^{\circ} \\
\hat{c}=109.33^{\circ}, \quad \frac{109.33^{\circ}}{16.364^{\circ}}=6.68 \Rightarrow \hat{c} \approx 7 \times 16.364^{\circ}=114.55^{\circ} .
\end{gathered}
$$

In the above calculations, the calculated numbers, are the central angles of the closest trilateral to that of the base that does meet Condition 2. The above calculations are used for the other proposed bases as well. In the next stage explained as follows, the new bases using the calculated angles are developed.

\section{Stage 3}

In order to draw a new base, a circle with the same radius of the circumscribing circle is drawn. Then, from the center of the circle, a number of lines with the calculated angles obtained from the previous stage are drawn, and their endpoints are connected together in order to find the desired polygon (Table 10).

\section{Stage 4}

In the final stage, the circumscribing circle is divided to the result obtained from stage 1 (22, 24, 26, 26 for triangle, quadrilateral, pentagon and octagon, respectively) (Table 11). It can be observed that all of the vertices of the new 
Table 10 The process of drawing a calculated polygon and displaying the amount of its error compared to the original base

Calculated trilateral

bases are precisely matched with the dividing points on the circle. The result shows that a Karbandi geometry can be accurately implemented in any of the new bases. Next, the distance by which the dividing points on the circle are connected to each other using Eq. (3) are obtained, are connected accordingly. Then, all the lines which are protracted out of the base or the ones inside the sousani are deleted to form the desired geometry of Karbandi (Table 11). The acquired Karbandi are the closest appropriate Karbandi to the primary bases.

It is clear that this method can be used to implement a Karbandi in all surrounded polygonal bases. As mentioned earlier, a slight level of error caused is completely normal even for symmetric and regular bases. For example, in (Pirnia and Bozorgmehri 2006: 13), there is a Karbandi which has been calculated and drawn for a $4 \mathrm{~m} \times 3 \mathrm{~m}$ rectangular base, yet its length and width are NOT exactly the mentioned sizes. Figure 11 displays the error level of the drawn Karbandi where the dashed line shows the original base and the continuous rectangle shows the base of the drawn Karbandi.

It should be noted that generally, by increasing the number of the sides of Karbandi, the level of error for the calculated base and consequently the level of error for the acquired Karbandi is reduced. The point is easily proved via Eq. (1). 
Table 11 The process of drawing Karbandi on the closest calculated polygons to the bases

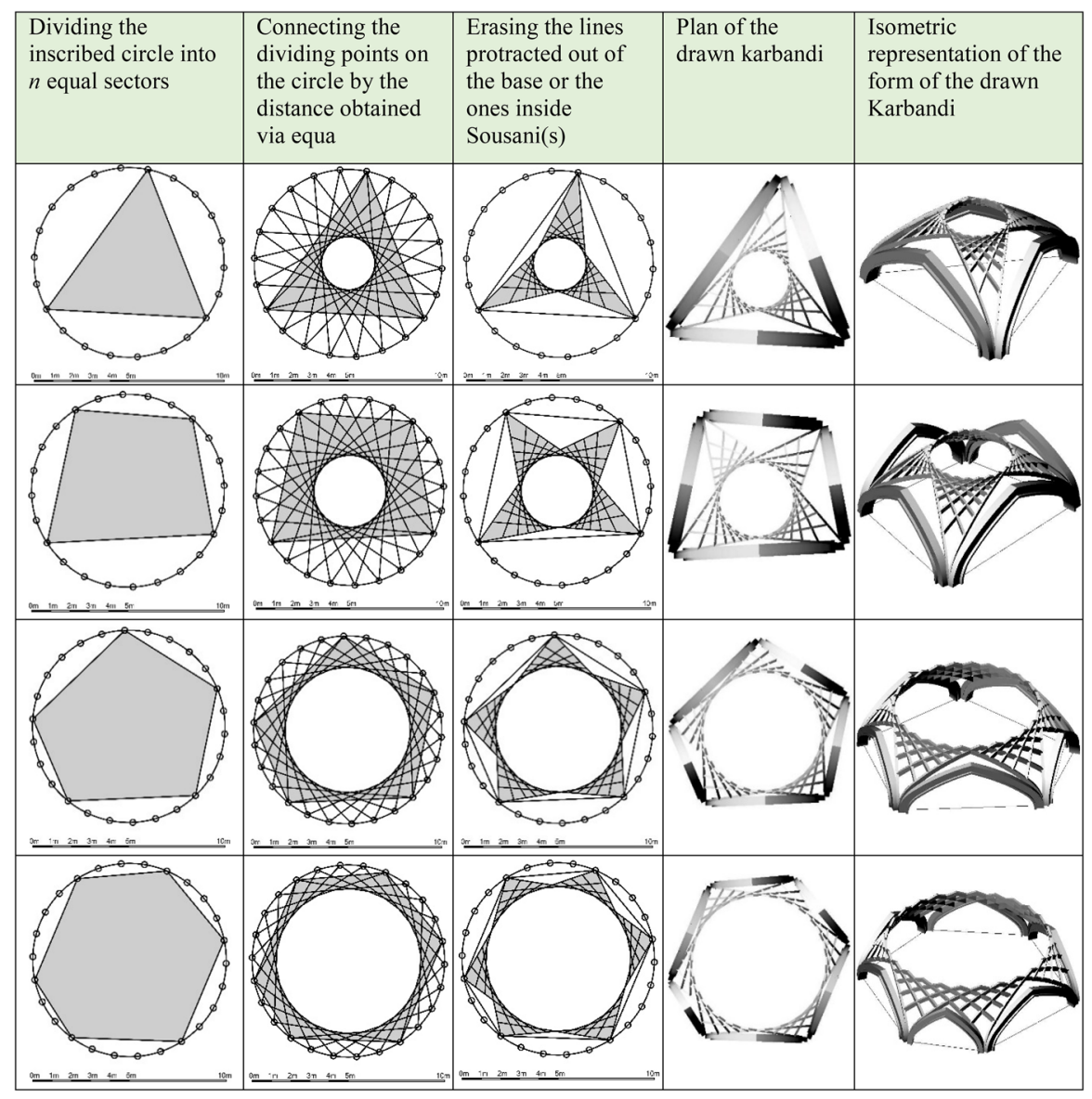

Fig. 11 The degree of error between the original base and the drawn base

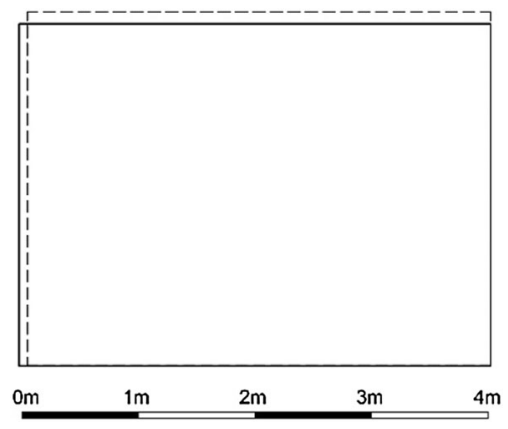




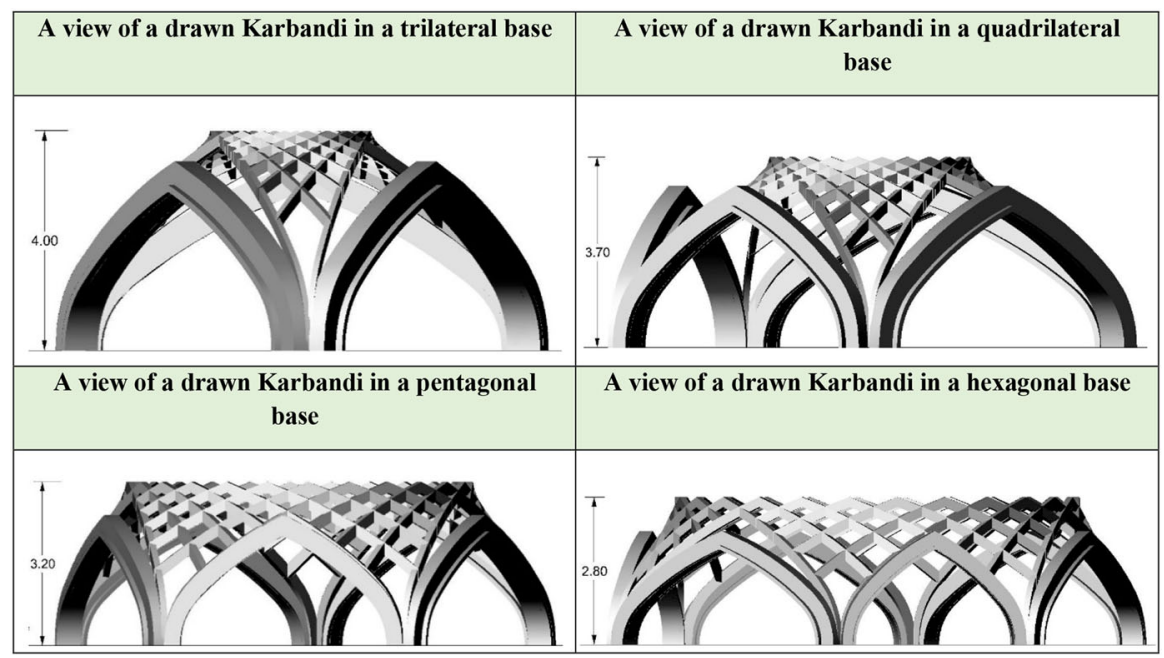

Fig. 12 A comparison of the height and chord (length) of the examples

By evaluating the examples, a number of variable formal properties are also observable. One of the most important of these qualities is the difference in the number of the sides of Karbandi. According to Eq. (5), it is clear that the number of the sides of a Karbandi is directly related to the perimeters of the base. The examples also show that as the number of sides of the bases increase, the number of the sides of Karbandi usually increase; this is due to the increase of the perimeter of the circumscribing circle (Table 11).

Another variable quality is the size of the shamseh, which is increased by increasing the number of the sides of the base. The cause of this is rooted in the length of the longest side of the base, in that as the number of its side $s$ increases, the length of the relative length of the longest side of the base decreases. As a result, the dividing points on the circle are connected to each other by a smaller distance, and consequently the size of diameter of shamseh increases (Table 11).

Changes in the height of the proposed examples is another point to be mentioned. The height of a Karbandi is directly related to the distance by which the dividing points on the circle are connected. Thus, when the mentioned distance is increased, the dimensions of the ribs of the Karbandi are also increased. By collating the previous point with this one, it becomes clear that the height of a Karbandi and the size of its shamseh are always mutually dependent. As evident in the examples, by increasing the dimensions of the shamseh, the height of the Karbandi is reduced (Fig. 12). 


\section{Conclusion}

This research aims at extending the usage of a precious heritage of Persian architecture in contemporary architecture by facilitating the creation of Karbandi in irregular geometries. The results of this paper shows that for an accurate implementation of an $n$-sided Karbandi in a number of polygonal bases, the following three conditions should be met: (1) the required base should be completely surrounded by a circle, (2) the angles formed as a result of connecting the vertices of the base to the center of the circle should be a multiple of the quotient of dividing $360^{\circ}$ by the number of the sides of the base $\left(\frac{360^{\circ}}{n} x\right)$, (3) the center of the circumscribing circle needs to be inside the base. Also, to calculate the suggested number of the sides of Karbandi $(n)$, in irregular and non-rectangular bases the following equation should be used:

$$
n=\left\{\begin{array}{c}
{[P-4]+1=[P]-3, \quad[P] \in \text { Odd }} \\
{[P-4]=[P]-4, \quad[P] \in \text { Even }}
\end{array} .\right.
$$

It is clear that in the above equation, by increasing the dimensions of the base, the number of the sides of Karbandi is also increased in order for the weight of the roof to be supported by more ribs. We eventually came up with a method that makes it possible to calculate and implement a Karbandi geometry on bases which only meet the first and third of the three conditions with the lowest level of error. Analyzing the drawn examples made it evident that by keeping the diameter of the circumscribing circle unchanged, an increase in the number of the sides of the base will result in the increasing of the size of its shamseh. Moreover, the height of a Karbandi and the size of its shamseh are always in an inverse relationship.

Acknowledgements All figures are by the authors.

\section{References}

Chenaghlou, M., A. Najad Ebrahimi, Y. Shahbazi, M. Kheirollahi, and A. Amjad Mohammadi. 2017. Structural Morphology of a Masonry Space Dome in Historical Tabriz Bazaar Complex. In: Interfaces: Architecture. Engineering. Science (Proceedings of the IASS Annual Symposium 2017, 25-28 September 2017, Hamburg, Germany).

Garofalo, V. 2016. The Geometry of a Domed Architecture: A Stately Example of Karbandi at Bagh-e Dolat Abad in Yazd. Nexus Network Journal 18(1) 169-195.

Helli, A. 1986. Gereh-ha va Qos-ha Dar Memari-ye Eslami (Gerehs and Arches in Islamic Architecture). Qom: Mehr (in Farsi).

Irving, Washington. 1931. The Alhambra. New York: Macmillan and Co.

Kaplan, Craig and David H. Salesin. 2004. Islamic Star Patterns in Absolute Geometry. ACM Transactions on Graphics 23(2): 97-119.

Koliji, Hooman. 2012. Revisiting the Squinch: From Squaring the Circle to Circling the Square. Nexus Network Journal 14(2): 291-305.

Lorzadeh, Hossein. 1979. Ehya-ye Honar Ha-ye Az Yad Rafteh (Revival of Forgotten Arts). Without official publisher (in Farsi).

Memarian, Gh. Hossein. 2012. Persian Architecture: Niaresh. Tehran: Naghme-ye No-andish (in Farsi). Mohammadian Mansour, S. and S. Faramarzi. 2011. Goune-shenasi Va Tadvin-e Sakhtar-e Hendesi-e karbandi dar Memari-e Iran (Typology and Formulation of Geometry structure of Karbandi in Persian Architecture). Honar-Ha-Ye-Ziba-Memari-Va-Shahrsazi 48(3): 97-109 (in Farsi). 
Nava'I, K and K. Haji Qassemi. 2011. Khesht-o Khial, An Interpretation of Iranian Islamic Architecture. Tehran: Soroush (in Farsi).

Pirnia, Mohammad-Karim and Bozorgmehri, Zohre. 2006. Hendese Dar Memari (Geometry in Architecture). Tehran: Sazman-e Miras-e Farhangi-ye Keshvar (Iranian Cultural Heritage Organization) (in Farsi).

Pour Ahmadi, Mojtaba. 2014. A Basic Method for Naming Persian Karbandis Using a Set of Numbers. Nexus Network Journal 16(2): 313-343.

Raeisi, M., M. Bemanian, and F. Tehrani. 2013. Rethinking the Concept of Karbandi Based on theoretical geometry, practical geometry and building function. Maremat \& Me'mari-e Iran 3(5): 33-54 (in Farsi).

Sharbaf, A. 2006. Gereh va Karbandi (Gereh and Karbandi). Tehran: Sazman-e Miras-e Farhangi-ye Keshvar (Iranian Cultural Heritage Organization) (in Farsi).

Stewart, Desmond. 1974. The Alhambra. Wonders of Man Series. New York: Newsweek Book Division.

Amir Amjad Mohammadi is an architect and an academic researcher who graduated from B. A. (Architecture Engineering, University of Tabriz, Tabriz, Iran) in 2015. The same year he succeeded to get admission to Tabriz Islamic Art University, which is amongst top 3 art universities of Iran, and received his master degree in 2017. Following the inception of master's degree, he started to conduct research in the area of geometrical and constructional optimization of architecture. His research interests are also in the area of computational design and fabrication.

Maziar Asefi holds a PhD degree in kinetic and transformable architecture from Liverpool University in the UK. He is an Associate Professor of Architectural Technology at Tabriz Islamic Art University in Iran, where he has also served as Graduate Program director in Architectural Technology. Maziar is an adjunct Faculty of Department of Architectural Science at Ryerson University in Canada since 2013. He has taught at Ryerson University, Liverpool University, Tehran University and IUST University. He was the winner the best researcher of the County Award in 2010 and 2017. Maziar taught a range of courses including design studio, transformable structures, prefabrication and mass-customization, advanced method of construction, and Islamic Architectural Structures. Maziar has registered two patents in the field of transformable architecture. He has published a book in kinetic architecture in English in 2010 and two books on architectural transformation and the relation between architecture and structure in Persian in 2017 and 2018. He presented over 100 papers in national and international journals, magazines and conference proceedings. He is a senior member in Iranian Construction Engineering Organization.

\footnotetext{
Ahad Nejad Ebrahimi is a practicing architect in Iran and an associate professor at the faculty of Architecture and Urbanism, Tabriz Islamic Art University, Iran. He holds a PhD degree in Architecture. During his fully funded study at the Faculty of Art and Architecture, Tarbiat Modares University of Tehran, he managed to publish several research outputs. Ahad's current research and teaching areas are as flows: Architectural Theory, Architectural Design, Urban Conservation, and Islamic Architecture. Ahad always made a great effort trying to support national heritage and conservation of architecture and urbanism. He also actively contributed to development of a program for conservation and restoration of historical building and urban areas in Iran.
} 